

\section{Die ganze Bandbreite der Radiologie}

Der 12. RadiologieKongressRuhr (RKR) vom 7. bis 8 . November 2019 steht wieder ganz im Zeichen der zielgerichteten radiologischen Fortbildung. Ein fortlaufendes Curriculum zur Diagnostik und Therapie aller Organregionen bietet umfassendes Know-how.

Organbezogene Veranstaltungsblöcke beispielsweise zu Abdomen, Thorax, Neuroradiologie, muskuloskelettaler Radiologie sowie Interventionen erlauben eine komprimierte Fortbildung. Die Referenten und Referentinnen werden aber auch Themen wie Mammadiagnostik und Uroradiologie in den Blick nehmen und aktuelle technische und berufspolitische Entwicklungen in der Radiologie beleuchten. Alle künftigen Ruhrkongresse werden nahtlos an das curricular aufgebaute Programm anknüpfen, sodass die Besuchenden sich gezielt weiterbilden können.

Zum 12. RKR feiert ein neues Session-Format Premiere: In „Meine schönsten Fehler“ berichten Fachleute über Fälle und diskutieren sie mit dem Publikum. Zum Programm gehören aber auch wieder Klassiker wie die Fit-fürden-Facharzt-Kurse, die Q1- und Q2-Kurse zum CT des Herzens und der Workshop für niedergelassene Radiologinnen und Radiologen. Das MTRA-Programm bietet ebenfalls eine große thematische Auswahl - auch hier ist vom Schülerprogramm bis zum themenfokussierten Kurs alles dabei.
Nach dem erfolgreichen Auftakt 2018 in Dortmund ist der RKR erneut in den Westfalenhallen zu Gast, die mit moderner Kongresstechnik, einem großzügigen Raumkonzept und einer guten Erreichbarkeit aus ganz Nordrhein-Westfalen punkten.

\section{SAVE THE DATE}

12. RadiologieKongressRuhr

7. bis 8. November 2019

Kongresszentrum Westfalenhallen Dortmund

www.radiologiekongressruhr.de 